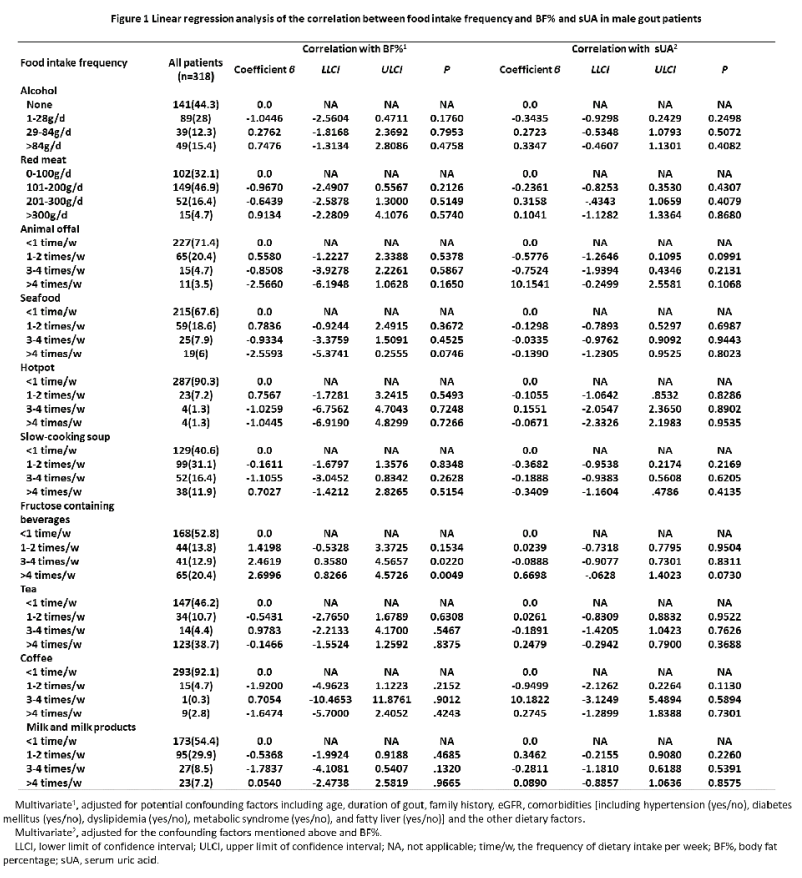

Conclusion: Our data show that body fat mediates the correlation between FCB consumption and SUA in male gout patients, which imply the importance of body composition assessment.

Acknowledgement: The present study was supported by Guangdong Natural Science Foundation, China (Grant no. 2014A030310086) to Qian-Hua Li.

Disclosure of Interests: None declared

DOI: 10.1136/annrheumdis-2019-eular.5774

\section{SAT0409 P2X7R PROMOTE THE ATTACK OF ACUTE GOUTY ARTHRITIS IN RATS FROM CLINICAL TO PATHOLOGY}

Xiaojuan Dai ${ }^{1}$, LI Xiangpei ${ }^{2}$, Jinhui Tao $^{2}$, Xuan Fang ${ }^{2}$, Yuan $\mathrm{Xia}^{2}$, LI Xiaomei ${ }^{2}$. ${ }^{1}$ The First Affiliated Hospital of University of Science and Technology of China, Rheumatology and Immunology, Hefei, China; ${ }^{2}$ The First Affiliated Hospital of University of Science and Technology of China, Rheumatology and Immunology, Hefei, China

Background: ATP may be the second causative signal for the onset of gout, which acts on P2X7R to regulate the development of acute gouty arthritis (1. P2X7R is the most relevant to the inflammatory response in the P2X family and is also an ATP-gated cation channel (2

Objectives: The aim of this study was to activate P2X7R signaling pathway through changes in extracellular ATP concentrations, leading to the development of acute gouty arthritis and the production of proinflammatory.

Methods: 120 male Sprague-Dawley rats were randomly divided into 3 groups: After establishment of acute gouty arthritis model, rats were given P2X7R agonist ATP, P2X7R inhibitor BBG and PBS, respectively. The clinical manifestations of the ankle joints were evaluated at $6 \mathrm{~h}, 12 \mathrm{~h}, 24 \mathrm{~h}$, $48 \mathrm{~h}$ and $72 \mathrm{~h}$ before the rats were sacrificed, and rat ankle synovial slices for H\&E staining. IL- $1 \beta$, IL- 6 and TNF- in the serum of rats were detected by ELISA kits.

Results: 1. P2X7R regulates the development of acute gouty arthritis: At $12 \mathrm{~h}$, the clinical scores of ATP group were significantly higher than those of BBG group and control group $(P=0.001,0.042)$, and the control group was higher than BBG group $(\mathrm{P}=0.034)$; At $12 \mathrm{~h}$ and $24 \mathrm{~h}$, the swelling index of ATP group was the most obvious than other two groups $(P=0.000,0.001 ; P=0.000,0.003)$, followed by control group $(\mathrm{P}=0.009,0.001) ; \quad$ Furthermore, there was a large infiltration of inflammatory cells in the synovial tissue of the right ankle joint of rats, at $12 \mathrm{~h}$, and $24 \mathrm{~h}$, the infiltration of mononuclear cell in ATP group was significantly higher than that in BBG group and control group $(P=0.000$ $0.007 ; P=0.000,0.001)$; The neutrophils infiltration in ATP group was the highest among the three groups at $24 \mathrm{~h}(\mathrm{P}=0.001,0.04)$, and the control group was higher than BBG group $(P=0.04)$. 2. P2X7R regulates pro-inflammatory cytokines production: At $24 \mathrm{~h}$, the level of IL$1 \beta$ in ATP group was significantly higher than BBG group and control group $(\mathrm{P}=0.001,0.003)$; At $6 \mathrm{~h}, 12 \mathrm{~h}$ and $24 \mathrm{~h}$, higher level of IL-6 in ATP group compared with BBG group and control group $(P=0.004,0.04$ $\mathrm{P}=0.000,0.002 ; \mathrm{P}=0.001,0.012$; ; The level of TNF- was obviously higher in ATP group than in BBG group and control group at $6 \mathrm{~h}$ and 24h ( $P=0.007,0.011 ; P=0.001,0.018)$, and $B B G$ group was lower than control group, but without statistically significant $(P>0.05)$.

Conclusion: Activation of P2X7R can significantly promote the attack of acute gouty arthritis and the production of IL-1 $1 \beta, \mathrm{IL}-6$ and TNF-, suggest ing that P2X7R affects the development of acute gouty arthritis and regulates the secretion of pro-inflammatory cytokines.

\section{REFERENCE}

[1] Tao JH, Zhang Y, Li XP. P2X7R: a potential key regulator of acute gouty arthritis. Semin Arthritis Rheum, 2013, 43(3): 376-380. (2) Hechler B, Gachet C. Purinergic receptors in thrombosis and inflammation[J]. Arterioscler Thromb Vasc Biol, 2015, 35:2307-2315

Disclosure of Interests: None declared DOI: 10.1136/annrheumdis-2019-eular.7147

\section{SAT0410 RADIOGRAPHIC EROSION SCORE IMPROVED WITH TARGETED URATE-LOWERING THERAPY IN A PROSPECTIVE GOUT VIETNAMESE COHORT}

Thomas Bardin ${ }^{1}$, Quang Dinh Nguyen², Khoi Minh Tran², Nghia Hieu Le², Duc Minh Do ${ }^{3}$, Valérie Bousson ${ }^{4}$, Pascal Richette ${ }^{1}$, Matthieu Resche-Rigon ${ }^{5} .{ }^{1}$ Hôpital Lariboisière, Rheumatology, Paris, France; ${ }^{2}$ Vien Gut Medical Center, FrenchVietnamese research center on gout and chronic diseases, HO Chi Minh City, Vietnam; ${ }^{3}$ University of Medicine and Pharmacy, Molecular Biology, Ho Chi Minh City, Vietnam; ${ }^{4}$ Hôpital Lariboisière, Radiology, Paris, France, ${ }^{5}$ Hôpital Saint Louis, Biostatistics, Paris, France

Background Urate deposition in joints of patients with neglected gout leads to destructive arthropathy, with subchondral bone erosion, bone construction, and late joint space narrowing. Gouty erosions are believed to improve under urate lowering drugs (ULDs), but their course has been little studied. We therefore performed a systematic prospective study of a Vietnamese cohort started on allopurinol at inclusion.

Objectives To characterize radiographic outcome of gouty erosions under serum urtae-targeted ULD

Methods Included patients had crystal-proven gout, eGFR $>60 \mathrm{ml} / \mathrm{min}$ and were not taking ULDs at inclusion. Allopurinol was progressively increased to reach EULAR- recommended uricemia targets and patients were prospectively followed-up, in particular with sequential hand and foot antero-posterior radiographs and MTP and knee ultrasound (US) scans. Joint erosions were scored according to a validated index 1 , double contour thickness was evaluated by a 4 pointscore. One index tophus per patient (hand or foot) was measured by US scan.

Results 100 patients $(99 \mathrm{M})$ with a mean age of $45.9+9.9$ years, and median gout duration of 8 years (range 0-33) were included. 91 had clinical tophi. Mean baseline uricemia was $492 \mu \mathrm{mol} / \mathrm{L}$. Median final allopurinol dose was $520 \mathrm{mg} /$ day. 71 patients had evidence of urate arthropathy at baseline, 68 of the feet and 23 of the hands (al but 3 with foot involvement). 1st MTPs were predominantly involved (44 and 35 patients on the right and left sides respectively). Urate arthropathy at baseline correlated with gout duration $(p=0.01)$, clinical tophi $(p=0.0007)$, maximum double contour thickness and index tophus US measurement $(p=0.03)$ by univariate analysis and with clinical tophi by multivariable analysis $(p=0.03)$. Uricemia target $<300 \mu \mathrm{mol} / \mathrm{L}$ was obtained in $63.6,67.8,70.6$, and $61.0 \%$ of patients at $3,6,12$ and 24 months respectively. Median radiological follow-up was 12 months (range 3-49). Mean radiological erosion score1 was 12.1 at baseline $(n=71)$, with a decrease of -0.7 at 6 months $(n=38$, 